Artículo de Investigación

A puntes del CEN ES

ISSN 0120-3053

Volumen 32 - №. 56

Julio - Diciembre de 2013

Págs. 37-52

\title{
La reforma tributaria de 1935 y el fortalecimiento de la tributación directa en Colombia
}

\section{The 1935 tax reform and the strengthening of direct taxation in Colombia}

Óliver M ora Toscano*

Fecha de recepción: 1 de febrero de 2013

Nueva versión: 8 de marzo de 2013

Fecha de aprobación: 15 de mayo de 2013

Docente asistente de tiempo completo, Escuela de Economía, Universidad Pedagógica y Tecnológica de Colombia (UPTC). El autor agradece los importantes y valiosos aportes hechos en la construcción de este artículo del profesor doctor Pedro Cézar Dutra Fonseca, docente del Programa de Postgrado en Economía de la Universidad Federal del Río Grande do Sul (UFRGS). 


\section{Resumen}

En este artículo se estudian los elementos más relevantes de la reforma tributaria propuesta y puesta en práctica por el presidente Alfonso López Pumarejo en 1935, durante su primera gestión de gobierno. La reforma fue implementada fundamentalmente mediante la Ley 78 de 1935 y complementada por medio de la Ley 63 de 1936. Se trata de uno de los importantes cambios realizados durante el período de la denominada "revolución en marcha". Uno de los elementos más relevantes de la reforma fue la consolidación y fortalecimiento de los impuestos directos en Colombia utilizando como instrumentos principales el incremento del impuesto a la renta y la creación de los impuestos al exceso de utilidades y al patrimonio. Los nuevos recursos fiscales obtenidos constituyeron una importante fuente de financiamiento del programa integral de reformas y políticas propuestas por el Gobierno lopista.

Palabras clave: historia económica, tributación directa, política económica, política tributaria.

Clasificación JEL: N36, H71, E61, E64

\section{Abstract}

This paper explores the most relevant elements of tax reform proposed and implemented by President Alfonso López Pumarejo in 1935, during his first term of government. The reform was implemented fundamentally through Act 78 of 1935 and complemented by means of Act 63 of 1936. It is one of the important changes made during the period of the so-called "ongoing revolution". One of the most relevant elements of the reform was the consolidation and strengthening of the direct taxes of Colombia using as principal instruments the increase of income tax and the creation of the taxes to the excess profits and estate tax. The new tax resources constituted an important source of financing of the comprehensive program of reforms and policies proposed by the government of President López.

Keywords: Economic history, direct taxation, economic policy, tax policy.

JEL Clasification: N36, H71, E61, E64 


\section{INTRODUCCIÓN}

En este artículo se analizan los aspectos más destacados de la reforma tributaria implementada durante la primera administración de Alfonso López Pumarejo. Esta reforma está consagrada principalmente en la Ley 78 de 1935 y complementada mediante Ley 63 de 1936. $\mathrm{El}$ aspecto más destacado de la misma fue la consolidación y fortalecimiento de la tributación directa en Colombia a través del aumento del impuesto a la renta y la creación de los impuestos al exceso de utilidades y al patrimonio.

Los nuevos recursos fiscales permitieron financiar un Estado que a partir de ese momento asume un papel más activo en la economía y la sociedad. De esta forma, el gobierno puede ampliar el gasto público en aquellos sectores fundamentales para el desarrollo tales como la educación, la salud, la higiene y el fomento económico.

En primer lugar son presentados los principales antecedentes normativos de la tributación directa en Colombia desde finales de la segunda década del siglo pasado. Posteriormente es analizada la situación fiscal existente a comienzos del primer Gobiernode López, situación queinfluenciará de manera importante en la decisión política de realizar la reforma. La escasez de recursos hace que el gobierno busque nuevas fuentes de financiamiento que permitan sufragar las principales necesidades financieras existentes en ese momento.

Después son estudiados los elementos centrales de la Ley 78 de 1935. Finalmente, a partir de los aspectos planteados en este artículo, se efectúa un balance general de la reforma tributaria implementada por el gobierno lopista. 


\section{ANTECEDENTES DE LA TRIBUTA- CIÓN DIRECTA EN COLOMBIA}

La tributación directa en Colombia se había tratado de implementar inmediatamente después de la independencia de España y, posteriormente, durante la primera República Liberal de mediados del siglo XIX (Ministerio de Hacienda y Crédito Público, 1936, p.24). En esos momentos no fue posible su realización por las precarias condiciones económicas existentes y el escaso desarrollo e integración de un mercado interno, además de la insuficiente voluntad política de las clases dirigentes (Ocampo \& Montenegro, 2007, p.343). La dinámica generada en la economía nacional por la expansión cafetera acontecida desde la segunda década del siglo XX, creó las condiciones que posibilitarían posteriormente la adopción definitiva de estos tributos.

Al final de la segunda década del siglo $\mathrm{XX}$, la política tributaria comenzó a experimentar un conjunto de cambios. Estos fueron posibles por los efectos que paulatinamente había comenzado a generar sobre la economía la expansión cafetera acontecida en ese momento, y el naciente proceso de crecimiento de la industria. En períodos anteriores esta política se fundamentaba en los tributos a los bienes de consumo masivo y el incremento de los impuestos al comercio exterior como el de aduanas.
En 1918 comienza una mudanza con la creación del impuesto a la renta mediante la Ley 56 de ese año, mudanza que se consolidará con la reforma de 1935. Esta norma procuró crear unas condiciones favorables para el crecimiento simultáneo del capital y el trabajo al introducir un sistema que gravaba diferencialmente las rentas según se originaran en uno o en otro. Sin embargo, este sistema tenía varias deficiencias que afectaron el nivel de recaudo. De esta manera, los ingresos fiscales provenientes de este tributo representaron solo el $2 \%$ de los ingresos públicos totales hasta 1927.

En ese año, 1927, fue promulgada la Ley 64, que estableció cambios importantes en el régimen legal de los impuestos directos. Hasta ese momento se habían aprobado otras disposiciones de menor importancia y que no introdujeron mudanzas profundas en la norma de $1918^{1}$. Esta ley estableció la tarifa progresiva y gradual, y precisó la diferencia entre renta bruta y renta líquida. Igualmente, la Ley 64 produjo un pequeño aumento en la participación del impuesto a la renta en el total de los tributos, mas sin conseguir el dinamismo alcanzado desde mediados de la década de los treinta. No obstante, esta norma representó un avance respecto a la legislación anterior.

En 1930, la segunda misión Kemmerer hizo una serie de recomendaciones sobre

\footnotetext{
Estas disposiciones fueron los decretos 1792 de 1922, 59 de 1924 y 802 de 1926 (Ministeriode Hacienda y Crédito Público, 1941, p.132).
} 
el impuesto a la renta, las cuales fueron recogidasen la Ley 81 de 1931. Esta norma unificó la administración de este impuesto a través de la dirección general de la Oficina de Rentas e Impuestos Nacionales y de los administradores de hacienda de la misma. Tal medida fue importante por cuanto la gestión de este tributo estaba descentralizada en las autoridades fiscales de cada municipio.

La misma misión Kemmerer caracterizó esta situación como problemática y conflictiva por las complicaciones vinculadasal origen partidista de los recaudadores municipales (liberal o conservador) (Ministeriode Hacienda y Crédito Público, 1941, p. 138-139). La Ley 81 también introdujo por primera vez en la legislación tributaria colombiana la retención en la fuente del impuesto a la renta $\left(\right.$ art. $\left.6^{\circ}\right)$. Esta ley es el antecedente normativo inmediato de la reforma efectuada en 1935 con la Ley 78.

\section{LA SITUACIÓN FISCAL EXIS- TENTE A COMIENZOS DE LA PRIMERA ADMINISTRACIÓN DE ALFONSO LÓPEZ}

A comienzos de la primera administración de López Pumarejo existía un importante déficit en las cuentas del Estado. Este desequilibrio comenzó a gestarse desde los años veinte cuando el gasto público experimentó un importante crecimiento. En ese momento la forma de financiar este gasto fue acudiendo al crédito externo, a los recursos originados en la indemnización pagada por Estados
Unidos por la pérdida de Panamá y al incremento de los ingresos provenientes del impuesto de aduanas y recargos (Kalmanovitz, 2003, p. 297-299). El aumento del recaudo por este impuesto se explicaba por la mayor disponibilidad de recursos para las importaciones como producto de la evolución favorable de los precios del café en la década de los veinte. Estos recursos representaban más del $50 \%$ del total de los ingresos públicos ordinarios.

La crisis económica de los años treinta tuvo como consecuenciauna disminución del comercio exterior que produjo la reducción de los recaudos por el impuesto de aduanas, principal fuente de financiamiento público. Esta situación generó un aumento del déficit (Ocampo $\&$ Montenegro, 2007, p. 342 y 343). Ante este contexto, los gobiernos de ese momento se esforzaron por consolidar un sistema tributario conformado por tributos internos, principalmente el de renta, para protegerse así de la inestabilidad del sector externo. A diferencia del de aduanas, el impuesto a la renta es menos vulnerable a los cambios bruscos del comercio internacional (Alviar \& Rojas, 1985, p.105).

El primer Gobierno de López Pumarejo requería encontrar fuentes de financiamiento para aumentar el gasto público, principalmente en rubros como salud y educación (Giraldo, 1994, p.30). Ante la difícil situación económica de los treinta y la disminución de los ingresos por los tributos al comercio exterior, una de las 
pocas alternativas existentes era el aumento de los impuestos a la actividad económica interna, es decir, los impuestos indirectos sobre el consumo y los directos sobre la renta y el capital.

El incremento de los tributos indirectos no era posible porque el mercado interno no estaba completamente desarrollado y formalizado y también por el costo político que implicaba establecer un gravamen al consumo en un contexto de crisis económica y social (Giraldo, 1994, p.30). Sobre este tema hacía referencia el ministro de Hacienda de la época, Gonzalo Restrepo, en las memorias entregadas al Congreso de la República:

Los impuestos indirectos tienen muchos y serios reparos porque gravando los productos, independientemente de la capacidad del contribuyente, imponen una carga onerosa a los ciudadanos pobres en quienes en definitiva se hace recaer (...). De ahí la tendencia de los países organizados hacia el establecimiento de impuestos directos, progresivos, porque con ellos se evita en gran parte el desplazamiento del gravamen y porque ofrecen la ventaja de afectar a los ciudadanos en proporción a su capacidad económica (Ministerio de Hacienda y Crédito Público, 1936, p.132).

Estas palabras del ministro reflejan la concepción del Gobierno lopista sobre la tributación, pues este considera los impuestos indirectos como regresivos y los directos como progresivos socialmente. Esta fue la tendencia predominante en la reforma tributaria aprobada posteriormente.

A los pocos días de su posesión como presidente de la República, López Pumarejo solicitó al Congreso la devolución del proyecto de presupuesto para 1935 presentado por la anterior administración. El propósito era introducir una serie de modificaciones que el gobierno consideraba como necesarias. El nuevo proyecto, entregado el 15 de noviembre de 1934, incluyó unos ingresos tributarios adicionales por cerca de $\$ 16$ millones, equivalentes más o menos al $25 \%$ de los gastos (Giraldo, 1994, p.31).

Entre los rubros que se modificaron se encontraba un aumento en los aportes generados por el impuesto predial nacional sobre los bienes inmuebles rurales y urbanos. Este rubro aportaría un total de \$5.2 millones. Este incremento generó la oposición del presidente de la comisión de reforma tributaria, el representante a la Cámara Carlos Lleras Restrepo, por cuanto competía injustamente con un tributo del mismo tipo cobrado por los gobiernos locales (Lleras, 1975, pp. 292-298).

También fue reactivado el impuesto de defensa nacional sobre el patrimonio que había sido creado durante la administración de Olaya Herrera. Igualmente fue incluido un impuesto de exceso de utilidades que gravaba los rendimientos de capital que fuesen 
superiores a cierta cantidad. La contribución de este rubro era estimada en \$1,2 millones (Giraldo, 1994, p.31).

Este paquete fiscal incluido en el nuevo proyecto de presupuesto no alcanzó a ser discutido en las sesiones ordinarias del Congreso. A pesar de convocar sesiones extraordinarias para comienzos del siguiente año, el presidente promulgó a finales de diciembre de 1934 dos decretos respaldados en el estado de excepción constitucional declarado con anterioridad con el objeto de financiar los gastos que implicó la guerra con el Perú. Estos decretos fueron el 2429 y el 2432 , mediante los cuales reajustaba el impuesto de renta y creaba el impuesto sobre exceso de utilidades.

Los dos decretos generaron mucha polémica por la forma como fueron promulgados y por su contenido. Finalmente, la Corte Suprema de Justicia, controlada por los conservadores, declaró la ilegalidad de esas normas. El contenido de estos decretos, ahora sin efecto legal, junto con otros elementos complementarios, fue presentado como un proyecto de ley de reforma tributaria el $1^{\circ}$ de agosto de 1935 durante las sesiones ordinarias del Congreso (Giraldo, 1994, p.32). Este era de composición completamente liberal, ante la abstención del Partido Conservador en las elecciones parlamentarias de abril. De esta forma, el Gobierno evitó la presentación de ese proyecto a comienzos de ese año cuando en el Senado y la Cámara existía todavía representación del conservatismo. Con un Congreso homogéneamente liberal, la propuesta de ley tenía ahora una mayor probabilidad de ser aprobada.

Después del proceso de negociación política, el Gobierno consigue finalmente en el Congreso la aprobación de la reforma tributaria a través de la Ley 78 de diciembre de 1935. El aspecto que más influyó en esta aprobación fue la existencia de importantes necesidades de recursos fiscales para financiar gastos urgentes en educación, higiene, salud y fomento económico. En ese momento la mayoría de miembros del Partido Liberal reconocían la existencia de estas necesidades, y si el Estado no era dotado de nuevos recursos para atenderlas, se incurriría en el riesgo de intensificar las dificultades económicas. Este riesgo se explicaba porque, ante la inexistencia de nuevas fuentes de financiamiento, no quedaba otra alternativa diferente que continuar acudiendo a la emisión para financiar el presupuesto (Giraldo, 1994, pp. 32 y 33). También existía el peligro de no poder contener el creciente descontento social existente en la época ante los negativos efectos de la crisis económica sobre el conjunto de la población.

En consecuencia, para resolver el problema fiscal y así dar continuidad a los distintos programas gubernamentales, era necesario hacerla reforma. La principal justificación gubernamental de las medidas tributarias contenidas en ella fue el papel redistributivo desempeñado por las finanzas públicas, justificación que 
era coherente con el discurso político del presidente. De esta forma, la Ley 78 de 1935 "era presentada como un proyecto en beneficio de los pobres, el cual, por lo tanto debía ser asumido por los ricos" (Giraldo, 1994, p.34). Otra de las razones esbozadas para la reforma era la retribución que las industrias debían entregar al conjunto de la sociedad por los beneficios obtenidos como producto de la protección aduanera proporcionada por el Estado durante muchos años (López, 1979, p.426) ${ }^{2}$.

\section{LOS ELEMENTOS CENTRALES DE LA REFORMA}

La reforma tributaria llevada a cabo mediante la Ley 78 de 1935, tomó como fundamento la estructura técnica de las anteriores normas sobre el tema, principalmente la Ley 81 de 1931 . Uno de los principales aspectos sobre los cuales se fundamentó la Ley 78, respecto a normas tributarias anteriores, fue "la precisión de los conceptos de renta, deducciones y exenciones" (Alviar \& Rojas, 1985, p.107).

En la reforma aprobada (Ley 78) se hace una definición más amplia, en comparación con la Ley 81, de las actividades empresariales y comerciales cuyas ganancias o beneficios forman parte de la renta bruta. Esta renta bruta, menos las deducciones contempladas en la norma, constituyen la renta líquida objeto del pago de impuesto sobre la renta.

En ese sentido, el artículo $1^{\circ}$ indica que la renta bruta comprende, además de los salarios y las rentas derivadas del trabajo, las ganancias, beneficios y rentas provenientes de profesiones, oficios, ocupaciones, negocios, comercio, transacciones sobre propiedades raíces o muebles, arrendamientos, dividendos y las rentas provenientes de capitales que hayan ingresado al patrimonio del contribuyente por donaciones, herencias, rifas o loterías.

El artículo $3^{\circ}$ establece por primera vez en la norma tributaria que no serán deducibles de la renta bruta, los gastos personales o de subsistencia del contribuyente y de su familia, las erogaciones hechas por concepto de reparaciones, construcciones y mejoras realizadas en cualquier propiedad. Estos ítems eran normalmente incluidos y generaban reducciones importantes del impuesto (de renta) que debía ser pagado.

En el numeral 4 del artículo $4^{\circ}$, la reforma indica que, con las excepciones previstas, toda persona natural, nacional o extranjera domiciliada en Colombia deberá ser gravada sobre su renta,

\footnotetext{
A partir de los años treinta aumentó la importancia económica y el tamaño del sector público nacional, centralizado y descentralizado, tanto en relación con el total del producto interno como respecto a las finanzas de los departamentos y los municipios. El mayor dinamismo del impuesto sobre la renta contribuyó a esta mayor relevancia del Estado central (Alviar \& Rojas, 1985, p.105). El aumento de los ingresos tributarios coincidió con la mayor participación del sector industrial en la economía nacional.
} 
cualquiera que sea el origen de ella, ya sea obtenida dentro o fuera del país. Este aspecto no estaba incluido en la anterior norma tributaria (Ley 81 de 1931).

En la Ley 78 desaparecen las deducciones de la renta bruta por pérdidas sufridas por las empresas durante el año gravable y no compensadas por seguros ${ }^{3}$. Estas deducciones estaban contempladas en la Ley 81. Igualmente permanece la deducción de la renta bruta de aquellas "cantidades invertidas por corporaciones, asociaciones o fundaciones exclusivamente en el sostenimiento de instituciones de asistencia pública o social, educación, organizaciones obreras, ligas cívicas y cámaras de comercio" (numeral 10, artículo $2^{\circ}$ ). Sin embargo, desaparece la reducción o exclusión del pago de impuesto a la renta de las corporaciones o asociaciones con fines exclusivamente religiosos contemplada en el artículo $4^{\circ}$ de la Ley 81. Es decir, la reforma tributaria de 1935 impone el pago de impuesto a la renta a la Iglesia.

En el numeral $7^{\circ}$ del artículo $4^{\circ}$ de la Ley 78 se aumentó la tarifa del impuesto a la renta del 7,5 al 17\%. Este incremento es respecto a la legislación anterior. El aumento del impuesto a la renta es coherente con la estrategia gubernamental de fomentar el crecimiento y desarrollo de la industria nacional. Aunque este era uno de los sectores gravados con este tributo, ese pago en cierto sentido retribuía al Estado la política proteccionista que permitía a los industriales disponer de un mercado interno todavía inexplotado y en proceso de crecimiento. Además ya existían para ese momento algunas obras de infraestructura que facilitaban la integración parcial de la economía nacional.

La Ley 81 de 1931 concedía en su artículo 10 una serie de exenciones en cantidades monetarias específicas en favor de las personas naturales. Estas exenciones reducían el monto del impuesto a la renta a pagar en la cuantía especificada en la norma. En la reforma se reducen esas cantidades monetarias y por ende las exenciones (artículo $8^{\circ}$, numerales 1,2 y 3 ). Sin embargo, la Ley 78 contempla por primera vez en la legislación tributaria el no pago del impuesto de renta a las personas que no tengan un patrimonio mayor de cierta cantidad, o renta distinta de un sueldo o salario cuando este no exceda también de cierto monto (artículo $8^{\circ}$, numeral 4). En ese sentido, en el artículo $9^{\circ}$ se reduce el monto de la renta bruta sobre la cual se está obligado a presentar declaración de renta.

La Ley 78 crea en el artículo 13 un impuesto adicional a la renta sobre el exceso de utilidades líquidas obtenidas en cada año gravable por las personas naturales o jurídicas gravadas con impuesto sobre la renta. La norma especifica que se entiende por utilidades

$3 \quad$ Las deducciones de la renta bruta disminuyen el valor de esta al momento de calcular la renta líquida objeto del pago de impuesto de renta. 
líquidas la diferencia entre la renta bruta y las deducciones y exenciones reconocidas legalmente. El exceso de utilidades se presenta cuando estas sobrepasan el $12 \%$ anual del valor del patrimonio (art. 17).

El impuesto al exceso de utilidades sería pagado entonces por quienes obtuvieran utilidades superiores a una determinada proporción de su patrimonio. Su adopción fue polémica por cuanto era considerado como un castigo a la eficiencia económica que permitía generar mayores utilidades. La razón más importante para la creación de este tributo fue el control a la evasión. En palabras del ministro de Hacienda de la época:

Juega más que todo (haciendo referencia al impuesto sobre exceso de utilidades), un importante papel de control para que no se burle el patrimonio, (. . .) porque si se oculta éste, con tal ocultación puede determinarse un exceso de utilidades, y si, por el contrario, por medios ingeniosos se infla el capital, para evitar exceso de utilidades, se causa entonces impuesto sobre el patrimonio (Ministerio de Hacienda y Crédito Público, 1936, p.29).

En el artículo 14, la reforma exime del pago de este impuesto adicional a las utilidades a las rentas provenientes de salarios, sueldos, pensiones, jornales y honorarios profesionales, y aquellas utilidades obtenidas de capitales invertidos en minas de oro, plata, platino y en las industrias del café y el banano. Por lo tanto, este nuevo impuesto iba dirigido principalmente al capital industrial.

Adicional al anterior tributo, la reforma tributaria crea un impuesto anual sobre el patrimonio, que es considerado como un impuesto complementario al de la renta. El impuesto al patrimonio sólo sería pagado por las personas naturales o jurídicas que paguen el tributo sobre la renta. Cuando estas personas no tengan renta gravable pero sí posean patrimonio, deben pagar este nuevo tributo. Al igual que en el caso del tributo a las utilidades, en el artículo 24 la norma exime del pago de este impuesto a los salarios, pensiones, jornales, honorarios profesionales y en general las rentas provenientes exclusivamente del trabajo. También los bienes pertenecientes a corporaciones o asociaciones que tengan fines exclusivamente de asistencia pública o social y los capitales invertidos en minas y en la industria cafetera y del banano.

La Ley 78 crea así dos impuestos complementarios al de la renta, estrechamente relacionados entre sí, el impuesto sobre el exceso de utilidades y el impuesto al patrimonio. Respecto a estos dos tributos, el ministro de Hacienda de ese momento expresaba, además, que:

quien quiera burlar la tasa adicional sobre exceso de utilidades, es cogido por la tasa complementaria sobre patrimonio (...); y quien quiera pagar menos patrimonio, disminuyéndolo artificialmente, se encuentra frente a la tasa adicional que grava el 
exceso (de utilidades) (Ministerio de Hacienda y Crédito Público, 1941, p.142).

Una de las dificultades más importantes respecto a la recaudación del impuesto a la renta había sido la imposibilidad del Estado de constatar la veracidad de las declaraciones. Esta problemática fue solucionada en parte a través de la reforma constitucional de 1936, la cual eliminó la reserva en los documentos privados del contribuyente y de esta forma autorizó el examen y constatación de los libros y archivos contables con fines impositivos. Esta medida permitió disminuir el fraude hasta ese momento existente en el contenido de las declaraciones (Ocampo \& Montenegro, 2007, p.347).

La reforma tributaria de 1935 significó una importante decisión política por parte del presidente López: transformar el impuesto a la renta en uno de los pilares de los ingresos fiscales del Estado (Ocampo \& Montenegro, 2007, p.347). Y en este aspecto radica precisamente su relevancia histórica. En efecto, entre 1932 y 1936 los ingresos del impuesto a la renta aumentaron de $\$ 1,5$ millones a $\$ 12,9$ millones, según puede ser observado en la Tabla1.

Tabla 1. Colombia: evolución del impuesto a la renta 1932-1938

\begin{tabular}{|c|c|c|}
\hline Años & $\begin{array}{c}\text { Nivel de } \\
\text { recaudo } \\
\text { (en millones } \\
\text { de pesos) }\end{array}$ & $\begin{array}{c}\text { Participación } \\
\text { en los ingresos } \\
\text { totales } \\
\text { (en porcentaje) }\end{array}$ \\
\hline 1932 & 1,5 & $4,2 \%$ \\
1933 & 1,7 & $3,9 \%$ \\
1934 & 2,0 & $3,7 \%$ \\
1935 & 4,3 & $7,0 \%$ \\
1936 & 12,9 & $17,4 \%$ \\
1937 & 15,5 & $16,8 \%$ \\
1938 & 18,2 & $20,1 \%$ \\
\hline
\end{tabular}

Fuente: Banco de la República (1994, p.133), Contraloría General de la República (1998).

Para ese mismo período esto representó un aumento en la participación de este tributo en el total de ingresos públicos del $4,2 \%$ al $17,4 \%$. Con este incremento, el impuesto de aduanas comenzó a disminuir en su importancia y contribución a las rentas públicas. En efecto, durante la segunda mitad de la década de los veinte su participación promedio fue superior al 60\%; ya en la segunda mitad de la década de los treinta esta participación media se redujo a menos del $38 \%$ (Tabla 2 ). 
La reforma tributaria de 1935 y el fortalecimiento de la tributación directa Ó liver M ora Toscano

Tabla 2. Colombia. Evolución del impuesto de aduanas 1932-1938

\begin{tabular}{|c|c|c|}
\hline Años & $\begin{array}{c}\text { Nivel de } \\
\text { recaudo } \\
\text { (en millones } \\
\text { de pesos) }\end{array}$ & $\begin{array}{c}\text { Participación } \\
\text { en los ingresos } \\
\text { totales } \\
\text { (en porcentaje) }\end{array}$ \\
\hline 1932 & 17,1 & $48,3 \%$ \\
1933 & 22,3 & $51,5 \%$ \\
1934 & 24,6 & $45,3 \%$ \\
1935 & 27,5 & $44,5 \%$ \\
1936 & 30,0 & $40,4 \%$ \\
1937 & 34,3 & $37,1 \%$ \\
1938 & 31,1 & $34,4 \%$ \\
\hline
\end{tabular}

Fuente: Banco de la República (1994, p.133), Contraloría General de la República (1998).

El presidente López consiguió aumentar el gasto público a una tasa anual promedio del $16 \%$ entre 1935 y 1938 y al mismo tiempo obtener un superávit fiscal hasta 1937. Esto se explica porque los ingresos corrientes superaron a los gastos excluyendo de éstos el gasto de la deuda. De esta forma, el gobierno aumentó el gasto público social mas también implementó un ajuste fiscal que permitió la estabilización de las finanzas públicas. La solución de la crisis fiscal a partir de 1935 permitió cambiar la orientación del presupuesto del nivel nacional. Este se orientó más hacia la atención de los sectores sociales, la expansión del mercado interno y el fomento económico.

La reforma tributaria de 1935 fue complementada en 1936 a través de la Ley 63. Esta norma, propuesta por el Gobierno lopista y finalmente aprobada por el Congreso, creó los impuestos sobre las herencias, asignaciones y donaciones.
También fueron establecidos nuevos mecanismos de control a la evasión.

Con las leyes 78 de 1935 y 63 de 1936, las finanzas públicas lograron depender menos del los recursos provenientes del crédito y los ingresos tributarios dependieron menos de las variacionesdel comercio internacional. La estructura tributaria adquirió un carácter más progresista, incrementó su participación dentro del PIB y revirtió la proporción de los impuestos directos e indirectos a favor de los primeros (Alviar \& Rojas, 1985, p.107)

\section{EL BALANCEDE LA REFORMA TRIBUTARIA}

La reforma tributaria de 1935 (Ley 78) y la Ley 63 de 1936 procuraron aumentar los mecanismos de financiamiento de un Estado que pretendía convertirse en el principal propulsor del crecimiento y el 
desarrollo económico y social (Molina, 1986, p.51). Desde la perspectiva del presidente, la reforma tributaria era fundamental para dotar al Estado de nuevos recursos que le permitiesen asumir un papel activo y dinámico en la economía y la sociedad colombiana. Esta permitiría, por lo tanto, financiar la intencionalidad de modernización y reformas procurada por el gobierno y, así mismo, constituía una expresión del acercamiento del sector lopista del liberalismo con los sectores populares.

A pesar de la existencia de una relativa favorabilidad a la reforma, ante el control absoluto del Congreso por parte del Partido Liberal, una revisión de las actas de discusión del proyecto de ley, contenidas en varios de los Anales de la Cámara de Representantes, demuestra que fueron realizadas varias modificaciones a la idea original del Gobierno. Por ejemplo, la propuesta original contemplaba la creación de un tributo a los giros sobre las exportaciones de café y un impuesto sobre las herencias. Como los cafeteros constituíanun gremio con importante influencia política y económica, se negoció con los congresistas la temporalidad del gravamen que afectaba a este sector, por la aprobación a cambio de otros tributos principalmente el de la renta. Así, el tributo a las exportaciones del grano no sería permanente y se autorizaba al gobierno a ceder a la Federación Nacional de Cafeteros parte de los recursos recaudados en aquellos casos de fuerte disminución de los ingresos de los productores, tal como aconteció en 1937.
Estas medidas fueron adoptadas en una ley específica y diferente a la del paquete tributario, la Ley 21 de 1935. En el caso del impuesto a las herencias, aunque este no fue aprobado en 1935, el gobierno consigue esa aprobación al año siguiente en un proyecto diferente y que constituiría posteriormente la Ley 63 de 1936.

En general, las modificaciones introducidas tanto en el Senado como en la Cámara al proyecto original del Gobierno, limitaron el alcance inicial del mismo. Estas limitaciones motivaron que en el diario El Espectador se escribiera que en general el Congreso "impuso un retroceso a la iniciativa gubernamental de los gravámenes directos, porque los jóvenes legisladores, elegidos para derrotar la APEN, se comportaron como para ganar el premio a la moderación" (1935, 18 de noviembre). En el caso particular del Senado, las divergencias entre las distintas corrientes del liberalismo obligaron al presidente de esta corporación a nombrar una comisión para solucionar estas diferencias y salvar así la reforma. Por lo tanto, el proceso de discusión de la reforma tributaria en el Congreso fue amplio e intenso.

Las medidas adoptadas en esta generaron la reacción en contra de varios sectores, principalmente los empresarios, los cafeteros y los terratenientes. Ellos aducían haber sido "arruinados" primero por la crisis económica y ahora por el Gobierno lopista (Kalmanovitz, 2003, p.357) y que el texto del proyecto finalmente aprobado era confiscatorio y 
afectaba por igual a todos los estratos sociales (Alviar \& Rojas, 1985, p.107). De hecho, la asociación que reunió a importantes sectores opuestos al Gobierno, la Asociación Patriótica y Económica Nacional (APEN), fue creada precisamente para oponerse específicamente a la reforma tributaria ${ }^{4}$. Posteriormente asumió la misma actitud con las otras reformas implementadas por el Gobierno. A pesar de la oposición, el presidente no cedió a estas presiones y finalmente consiguió la aprobación de la nueva norma tributaria. Con ella, el Estado prácticamente duplicó su capacidad financiera con tributos pagados fundamentalmente por aquellas personas de altos ingresos económicos.

\section{CONCLUSIONES}

En general, la Ley 78 de 1935 "marcó un hito en la historia tributaria del país" (López, 1998, p.157). Uno de los propósitos era consolidar los principios democráticos y de progresividad de la tributación mediante el aumento de los impuestos directos y transformar la mentalidad existente en los contribuyentes, según la cual la tributación constituía una costosa exacción que implicaba la pérdida de recursos económicos (López, 1998, p.157).
Desde 1918, cuando apareció por primera vez el impuesto a la renta, las autoridades económicas se encontraron con varios obstáculos y resistencias que hacían compleja la efectiva recaudación del tributo. Dificultades que se explican por varios factores. Este impuesto afectaba los intereses de los grupos de población de mayores ingresos y por tanto era rechazado por ellos. Los procedimientos de información y pesquisa necesarios para hacer el cálculo de la tarifa enfrentaron la abierta oposición de los afectados. Además había una escasa preparación técnica entre los funcionarios de Hacienda encargados de realizar las respectivas liquidaciones y, finalmente, existía en general una práctica de evasión y ocultación de bienes que generaba unas bajas recaudaciones del tributo. Por esta razón el Gobierno lopista intentó eliminarestos obstáculos y aplicar los principios liberales de justicia social a la estructura tributaria existente (López, 1998, p.157).

Después de la promulgación de la Ley 78 de 1935 , las autoridades gubernamentales pretendieron cambiar la concepción de los impuestos como una pesada carga y un costo para los recursos privados, por la de un instrumento de estímulo al desarrollo

\footnotetext{
4 El periódico El Tiempo (1935, 8 de marzo), manifestó que la APEN era una organización que reunía varios importantes sectores opuestos a las políticas implementadas por López Pumarejo durante su primera administración. Esta fue fundada en marzo de 1935. En su manifiesto de creación señalaba que uno de sus primeros objetivos sería impedir que prosperan en Colombia aquellas ideas tendientes a la abolición de la propiedad privada y la desaparición del capital, haciendo así referencia a las reformas constitucional y tributaria propuestas por el gobierno lopista. Sobre el aspecto tributario, esta asociación proponía hacer una revisión del sistema impositivo con fundamento en un criterio científico y no en uno basado en la idea de la revolución social y los odios de clase.
} 
de las actividades económicas, tanto públicas como privadas, y con beneficios para todo el conjunto de la sociedad. Razón por la cual el Gobierno implementó una importante actividad de educación y concientización entre la población para intentar demostrar los aspectos positivos de la tributación. A los comerciantes les recalcó la importancia de la inversión pública en aspectos fundamentales como puertos, ferrocarriles y carreteras, a los industriales les mostró las bondades de la protección proporcionada por la tarifa aduanera, a los inversionistas las efectos positivos de la política tributaria sobre el mantenimiento de una moneda sana y estable $\mathrm{y}$, finalmente, a los trabajadores $\mathrm{y}$ a la población en general, el Gobierno intentó asociar la reforma con la defensa de sus intereses y derechos a partir de unos principios de equidad y progresividad (López, 1998, p. 157 y 158).
La política y reforma tributaria aplicadas durante el primer Gobierno de López Pumarejo "ha constituido el único intento en el presente siglo (siglo XX) de integrar acción instrumental con acción educativa, tendiente a aumentar el recaudo con resultados ampliamente positivos" (López, 1998, p.158). Sin embargo, esta intencionalidad no consiguió eliminar por completo la oposición y críticas de aquellos sectores más afectados, los grupos de ingresos monetarios más altos, empresarios, terratenientes y comerciantes. Desde una perspectiva histórica, la reforma constituyó uno de los esfuerzos más importantes en el siglo XX por establecer una estructura tributaria progresiva y que contribuyese en el financiamiento de un Estado que promoviese el crecimiento y el desarrollo endógeno.

\section{Referencias}

Alviar, O. \& Rojas, F. (1985). Elementos de finanzas públicas en Colombia. Bogotá: Temis.

Banco de la República. (1994). Principales indicadores económicos 1923-1992. Bogotá: Banco de la República.

Congreso de Colombia. (1931, diciembre). Ley 81 relativa al impuesto sobre la renta. Bogotá: Imprenta Nacional.

Congreso de Colombia. (1935, diciembre). Ley 78, por la cual se reforman las disposiciones vigentes del impuesto sobre la renta, se aumenta la tarifa, se establecen unos impuestos adicionales y se suprimen otros. Bogotá: Imprenta Nacional.

Congreso de Colombia. (1936b, marzo). Ley 63 de 1936, por la cual se organizan los impuestos sobre la masa global hereditaria, asignaciones y donaciones. Bogotá: Imprenta Nacional.

Giraldo, C. (1994). Estado y hacienda pública en Colombia 1934-1990. Bogotá: Contraloría General de la República. 
Kalmanóvitz, S. (2003). Economía y nación: Una breve historia de Colombia. ( $2^{\mathrm{a}}$ ed.). Bogotá: Norma.

López, A. (1979). Obras selectas. J.M. Eastman (comp.). Bogotá: Imprenta Nacional, Cámara de Representantes de Colombia.

López, H. (1998). Tributación y falta de legitimidad en Colombia. En Cuadernos de Economía, Universidad Nacional de Colombia, (28), 151-162.

Lleras, C. (1975). Borradores para una historia de la República Liberal. Vol. 1. Editorial Nueva Frontera, Bogotá.

Ministerio de Hacienda y Crédito Público (1936): Memoria de hacienda, 19351936. Bogotá: Nueva Frontera.

Ministerio de Hacienda y Crédito Público. (1941). Memoria del ministro de Hacienda y Crédito Público al Congreso Nacional de 1941. Bogotá: Imprenta Nacional.
Molina, G. (1986). Las ideas liberales en Colombia. De 1935 a la iniciación del Frente Nacional ( $7^{\mathrm{a}}$ ed.). Bogotá: Tercer Mundo.

Ocampo, J. A. \& Montenegro, S. (2007). Crisis mundial, protección e industrialización. (2 ${ }^{\mathrm{a}}$ ed.). Bogotá: Norma.

Presidencia de la República de Colombia. (1934b, diciembre). Decreto 2429, sobre la modificación del impuesto de renta. Bogotá: Imprenta Nacional.

Presidencia de la República. (1934c, diciembre). Decreto 2432, sobre la creación del impuesto sobre exceso de utilidades. Bogotá: Imprenta Nacional.

Urrego, M. (2005). La revolución en marcha en Colombia (1934-1938): una lectura en perspectiva latinoamericana. Michoacán, Méjico: Universidad Michoacana de San Nicolás de Hidalgo, Instituto de Investigaciones Históricas. 